\title{
321 - Association between quality of life and anxiety, depression, and comorbid anxiety and depression in people with dementia in nursing homes: A 12-month follow-up study
}

Alka R. Goyal ${ }^{1}$, Sverre Bergh ${ }^{2,3}$, Knut Engedal ${ }^{3}$, Marit Kirkevold ${ }^{1,4}, \varnothing_{\text {yvind Kirkevold }}{ }^{2,3,5}$

${ }^{1}$ Faculty of Health Sciences, Department of Nursing and Health Promotion, Oslo Metropolitan University, Norway

${ }^{2}$ The Research Centre for Age Related Functional Decline and Diseases, Innlandet Hospital Trust, Norway

${ }^{3}$ Norwegian National Advisory Unit on Aging and Health, Vestfold Hospital Trust, Norway

${ }^{4}$ Department of Nursing Science, Institute of Health and Society, University of Oslo, Norway

${ }^{5}$ Norwegian University of Science and Technology (NTNU) in Gjøvik, Faculty of Health, Care and Nursing, Norway

Background: Anxiety and depression are highly prevalent among people with dementia (PWD), and has many negative outcomes, such as increased behavioral problems, decreased ability to live independently, and increased risk of nursing home placement, which may affect the person's quality of life (QoL). Many cross-sectional studies have investigated factors associated with QoL in PWD in nursing homes, but few longitudinal studies have investigated how anxiety and depression affect the course of QoL of PWD in nursing homes. This study aimed to explore the association between QoL and anxiety, depression, and comorbid anxiety and depression in PWD in nursing homes at a 12-month follow-up.

Methods: In all, 298 PWD $\geq 65$ years old from 17 Norwegian nursing homes were assessed with Norwegian version of the Rating Anxiety in Dementia scale (RAID-N). Anxiety was defined as RAID-N score $\geq 12$. QoL was assessed by Quality of Life in Late-Stage Dementia (QUALID) scale. Depression was assessed by the Cornell scale of depression in dementia (CSDD), defined as CSDD score $\geq 10$. The assessments were made at baseline and after a mean follow-up period of 350 days (SD 12.3). A growth mixture model revealed two distinct trajectories of QUALID scores. Participants' characteristics and related risk factors associated to QUALID score trajectories were analyzed by logistic regression models.

Results: In trajectory group 1 (206 participants), a lower proportion of participants had anxiety, and a more stable and better QoL, compared to trajectory group 2 (92 participants) with a higher proportion of participants with anxiety and poorer QoL at a 12-month follow-up. Participants with comorbid anxiety and depression had the worst QoL than the participants with only anxiety or only depression. Adjusted multivariate logistic regression analysis revealed that more severe impairment in activities of daily living, presence of anxiety, depression, agitation, and use of antipsychotics at baseline, were associated with belonging to the trajectory group with poor QoL.

Conclusion: It is important among nursing home personnel to increase awareness of and skills in identifying anxiety and depression, and to initiate adequate management to enhance Qol of PWD in nursing homes.

Note: The material was published in September 11, 2018 in Plos one. 